\title{
Structural and EELS studies on Doped Carbon Nanostructures for Cold Field Emission
}

Rongrong Wang ${ }^{1,2}$, Raul Arenal ${ }^{1,3}$, Aurélien Masseboeuf $f^{2}$, David Neumeyer ${ }^{2}$, Marc Monthioux ${ }^{2}$ and Alejandro Lopez-Bezanilla ${ }^{4}$

1. Laboratorio de Microscopias Avanzadas, INA Zaragoza, Zaragoza - Spain.

2. CEMES - CNRS, Toulouse - France.

3. Fundacion ARAID, Zaragoza - Spain.

4. Materials Science Div., Argonne National Lab. Illinois, USA.

Along with the development of cold field emission technology, more and more microscopes equipped with cold field emission gun (C-FEG) are applied in scientific research. Thanks to the lower working temperature, the service time of emitter has been extended. In the meantime, this technology allows to emit electrons from smaller emission radius compared to thermionic emission and Schottky emission [1]. Based on this property, higher current density, higher brightness, better special coherence and lower energy distribution of the emission can be expected, which also means that a better electron beam can be obtained [2]. For the further improvement of cold field emission, new emitter materials have been explored. As a rolled-up graphene sheet, carbon nanotubes (CNTs) have the similar property, for instance, low work function, high mechanic performance, high melting point, high carrier mobility [3-4]. In addition, CNTs have small apex radius curvature for its quasi-one-dimensional character at nanoscale. Therefore, CNTs are capable of working under the operating condition of cold field emission [5]. Furthermore, carbon nanocones are considered as candidate as well [6]. Significant improvements of such kind of carbon nanocones are demonstrated by replacing the regular tungsten tip in transmission electron microscope [7]. For a higher performance of the cold field emission application, the electronic band structure of carbon nano-object can be modulated through the introduction of heteroatoms and a lower work function can be expected [8,9]. Our work is devoted to the doping of these carbon nanostructures by nitrogen and/or boron, and to the evaluation of these doped carbon nano-objects by a characterization for the cold field emission application.

We started our doping study from the CNTs. The doping of CNTs was carried out in a tubular furnace from $1300{ }^{\circ} \mathrm{C}$ to $1500{ }^{\circ} \mathrm{C}$ under different atmosphere. Doped CNTs were characterized by transmission electron microscopy: high resolution imaging (HRTEM) and spatially resolved electron energy loss spectroscopy (EELS) in scanning (STEM) mode. Those studies were developed using 2 different aberrations corrected TEMs (FEI-Titan), working at low acceleration voltage $(80 \mathrm{kV})$ in order to prevent nanotubes from the damage caused by electronic irradiation. In addition X-ray photoelectron spectroscopy (XPS, Kratos Analytical) has also been carried out. By these characterizations, structural modification after thermal treatment can be studied and the incorporation of hetero elements can be identified at local and macro scale. The pristine CNTs are compared with doped CNTs (fig. 1), this comparison illustrates that there is non-significant structural modification after the thermal treatment. The formations of C-B bonding and C-N bonding are identified in the spectra at macro scale by XPS (fig. 2 (a)). The presence of boron and nitrogen inside CNTs at local scale is confirmed by EELS (fig. 2 (b)) [10]. 


\section{References:}

[1] A. V. Crewe et al., The Review of Scientific Instruments 39 (1968) p. 576.

[2] N. De Jonge et al., Nature 420 (2002), p. 393.

[3] J. W. Mintmire, B. I. Dunlap and C. T. White, Physical Review Letters 68 (1992) p. 631.

[4] J.N. Coleman et al., Carbon 44 (2006) p. 1624.

[5] J.-C. Charlier et al., Nano Letters 2 (2002) p. 1191.

[6] L. Ronald and M. Montioux, Nature 385 (1997) p. 211.

[7] F. Houdellier et al., Carbon 50 (2012) p. 2037.

[8] P. Ayala et al., Reviews of modern physics 82 (2010) p. 1843.

[9] R. Arenal, X. Blasé and A. Loiseau, Advances in Physics 59 (2010) p. 101.

[10] This work was supported by the project ANR LASCAR (ANR-13-BS04-0007), the project ESTEEM2 (Integrated Infrastructure Initiative - I3, Grant Agreement 312483), the Spanish MINECO (FIS2013-46159-C3-3-P), and international associated laboratory TALEM (CNRS - U. of Zaragoza). TEM studies were developed in the Advanced Microscopy Laboratory (LMA) of Institute of Nanoscience of Aragon (INA) - U. of Zaragoza (Spain).
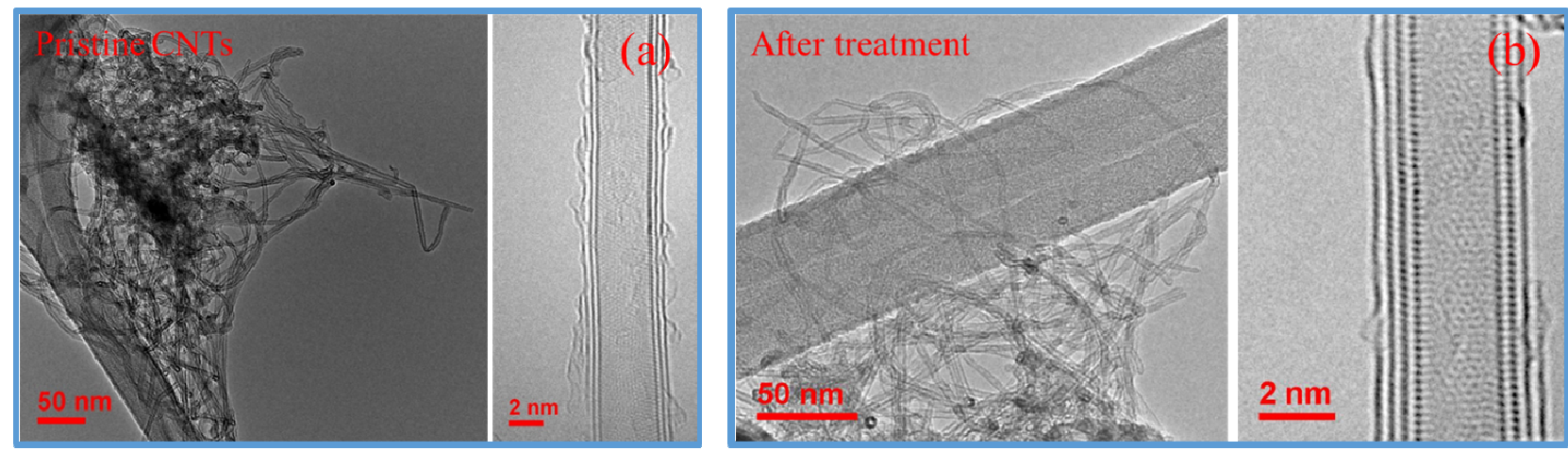

Figure 1. Structural comparison between pristine (a) and doped CNTs (b) at different magnification.
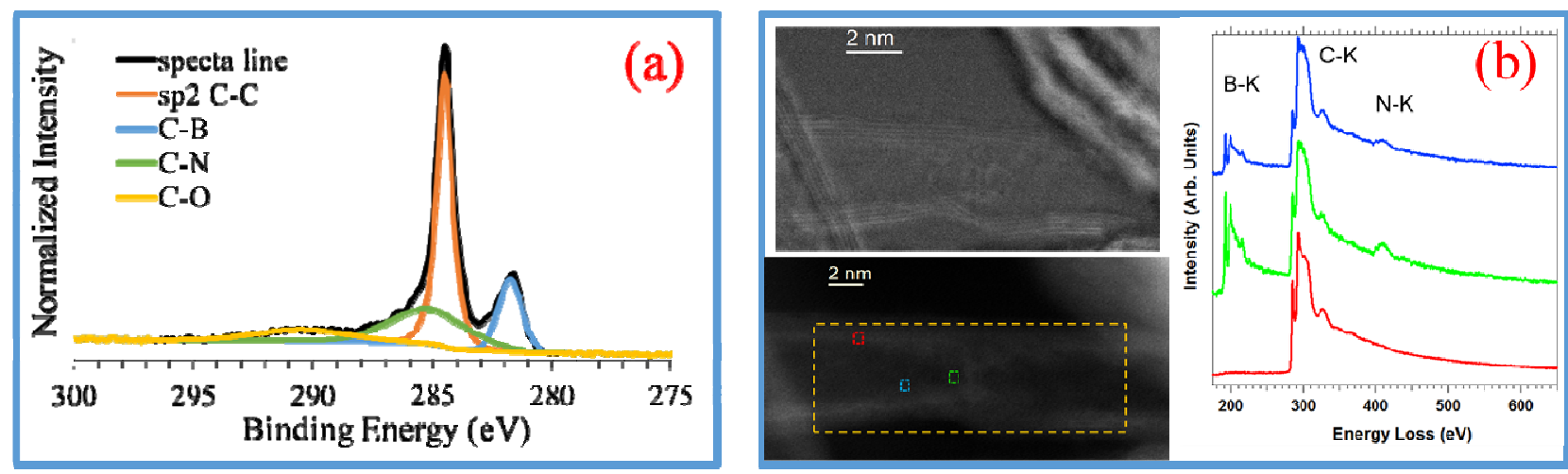

Figure 2. (a) XPS spectra of carbon C1s binding energy. (b) Selection of EEL spectra extracted from the spectra image; blue, red and green spectra correspond to the pixels outlined in blue, red and green in bottom left image. 